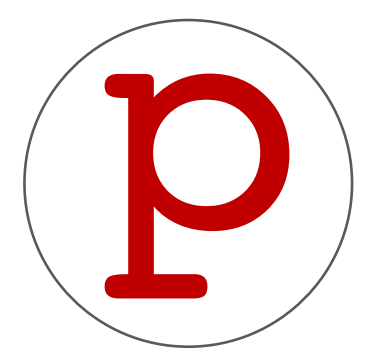

polis isemiee

$$
\mid
$$

I

2020 


\section{pol isemie}

I

\section{0}

Direzione

Stefano Milonia (University of Warwick)

Comitato scientifico

Niccolò Scaffai (Università degli Studi di Siena)

Carlo Pulsoni (Università di Perugia)

Luigi Marinelli (Università di Roma Sapienza)

Giulia Bassi (Università degli Studi di Siena)

Mario Cianfoni (Università di Roma Sapienza)

Costantino Turchi (Università di Roma Sapienza)

\section{Redazione}

Mattia Caponi (Università di Roma Sapienza)

Stefano Bottero (Università di Roma Sapienza)

Samuele Maria Visalli (Università di Roma Sapienza)

Giuseppe Giorgio Tranchida (Alma Mater Studiorum Università di Bologna)

Arianna Saggio (Università di Roma Sapienza)

Giulia Boitani (University of Cambridge)

Giulia Greco

Alessandra Frustaci

Polisemie è una rivista annuale pubblicata dalla University of Warwick Press.

Gli articoli pubblicati nella sezione Saggi sono sottoposti a double-blind peer review.

Licenza Creative Commons - Attribuzione (CC-BY 4.0).

ISSN: 2634-1867

DOI: 10.31273/polisemie.v1

Copertina: Alessia Provinciali 


\title{
ROBERT VISCUSI: INCONTRO CON L'AUTORE
}

\author{
Bologna, 11 ottobre 2012
}

Erica Verducci

In occasione dell'incontro "The stories disintegrate you like waves", organizzato dalla casa editrice abrigliasciolta di Varese in collaborazione con la libreria Le Moline di Bologna, conoscevo Robert Viscusi per la prima volta e avevo la possibilità di ascoltare le sue preziose parole in merito alla sua opera creativa. In questa circostanza l'autore presentava il terzo volume di Ellis Island, in uscita in quel periodo e coincidente con la stesura della mia tesi di laurea Robert Viscusi e l'eredità italoamericana. $\mathrm{Mi}$ interessavano allora gli elementi autobiografici presenti nel romanzo Astoria e confluiti poi nella raccolta Ellis Island, opere che erano state considerate entrambe narrazioni personali atipiche. In quanto autobiografia romanzata, Astoria mi imponeva la necessità di distinguere la componente biografica vera e propria da quell'elemento di finzione che la faceva uscire dal suo presupposto cronachistico. Fu quindi fondamentale partecipare all'appuntamento con l'autore e prestare attenzione alle storie e agli aneddoti che raccontava, storie che erano già presenti nel romanzo e altre che finalmente davano una spiegazione ad elementi narrativi per me incomprensibili perché non avevano - almeno apparentemente - connessione con le vicende personali note di Viscusi.

A differenza di Ellis Island, che narra di un viaggio ideale, a tratti nebuloso, Astoria si svolge in luoghi ben definiti. Luoghi che si configurano anch'essi come tappe di un viaggio - il famoso "viaggio al contrario" dall'America all'Italia - ma che vengono descritti e indagati come spazi funzionali alla ricostruzione di un'identità e per questo imprescindibili. Non si tratta semplicemente di spazi reali di vita vissuta, ma soprattutto di contesti emotivi che, per questa ragione, sono diventati pretesti narrativi, spazi da cui sviluppare un racconto.

I luoghi di Astoria sono tre grandi rappresentanti di un'epoca, ma anche di una storia in atto. Tutti quanti - Astoria, quartiere del Queens, Parigi e Roma - si incontrano nel presente dell'autore e scandiscono la narrazione del suo viaggio reale verso l'Italia; sussistono in quanto "stazioni" in cui si svolgono vicende 
diverse. Si potrebbero ugualmente leggere come "stadi” dell'esistenza dell'autore, così come fasi emotive, luoghi da cui giungono memorie e personaggi:

Astoria, in real life, is the name of an immigrant neighborhood in North Queens. Its faded glories don't amount to much and never did, especially when you see them against the strict laws of immigrant life - the narrow doorways, the pinched purses, the carefully graduated ambitions. So the professor's pretences have a wistful music about them. He is like a child who announces that some broken water pipe is a miraculous fountain. He makes Paris and Rome colonies of Astoria, as if his mother's childhood, bloody and bitter as it was, had really been an empire, as if he had actually been its heir. ${ }^{1}$

Viscusi espone i fatti facendo slittare continuamente il discorso da un piano temporale all'altro, suscitando così nel lettore una sorta di incertezza e di esitazione, poiché il momento narrativo a cui partecipiamo non permette di distinguere subito il passato dal presente. Le vicende della madre in particolare sembrano rimanere sospese nel tempo: «She takes the girl and the boy into the glass vestibule, an acquarium of mildew and milk bottles in metal crates. She leads up the stairs. Forty years later she is dying, trying to spell with her eyelids, "My chest hurts" ». ${ }^{2}$ C'è però la volontà di commemorare quel passato italiano che riunisce le sorti di tutti i personaggi in episodi che non possono essere definiti semplicemente "nostalgici", dal momento che ora rappresentano interamente il presente dell'autore:

$\mathrm{He}^{3}$ had four grandparents. His father's mother. His father's stepfather. They lived in Sunnyside. His mother's mother. His mother's father. These lived in Astoria, another

\footnotetext{
${ }^{1}$ Robert Viscusi, Astoria, Toronto, Guernica, 2003, p. 8 (trad. it. Cava de' Tirreni, Avagliano, 2003, p. 6: «Astoria, nella vita reale, è il nome di un quartiere di immigrati nella parte settentrionale del Queens, a New York. Le sue glorie sbiadite non hanno mai rappresentato molto, specialmente quando le osserviamo sullo sfondo delle severe leggi che regolano la vita degli emigranti, le ristrettezze, i cordoni della borsa tenuti serrati, le ambizioni attentamente graduate. Cosicché le pretese del professore hanno il suono di una musica malinconica. È come un bambino che annuncia che una conduttura d'acqua rotta è una fontana miracolosa. Fa di Parigi, e Roma, colonie di Astoria, come se l'infanzia della madre, dannata e amara come fu, fosse stata in realtà un impero, come se egli ne fosse stato davvero l'erede»).

${ }^{2}$ Ivi, p. 30 (trad. it. p. 29: «Lei conduce la bambina e il bambino nell'ingresso a vetrate, un acquario pieno di muffa e di bottiglie di latte in recipienti metallici. Sale per prima le scale. Quarant'anni più tardi, sta morendo, cercando di sillabare con le palpebre 'Mi duole il petto' »).

3 "He" [lui] è il nostro protagonista, è Robert che parla di se stesso in terza persona. Prima di leggere per la seconda volta il romanzo avevo dato per scontato che il gioco di parole l'avesse inventato l'autore riflettendo sulle molteplici possibilità di significato date dalla parola Astoria; adesso invece mi chiedo se non siano state proprio le ingenue storpiature di questo nome da parte dei nonni ad aver innescato la riflessione sul termine in questi continui rimandi. L'"io" ricomposto dell'autore
} 
part of Queens. They called it l'Astoria, which was unconsciously a complicated pun that meant history and also meant abistory. Since they did not make anything of it, he was going to have to do so. Astoria, l'Astoria, was a persistent trace. He kept finding it all over Paris. But begin at the beginning. ${ }^{4}$

Si può notare immediatamente come la parola "Astoria" si presti a numerosi giochi di parole e sovrapposizioni di concetti. Viscusi ci fa partecipi di questo processo già dalla primissima pagina, anticipando ciò che accade nel romanzo con le espressioni "Astoria", "L’Astoria", "La Storia", in un continuo rimando di significati. Astoria, il quartiere italiano del Queens, l'Astoria, cioè la sindrome di cui crede di essere affetto l'autore (ma, attenzione, a-Storia può essere inteso anche come "assenza di storia"), e la Storia, in riferimento alla storia di tutti gli emigrati italiani in America (e, volendo, storia dei popoli migranti e, quindi, dell'umanità intera) sono le diverse facce di quell'identità che Viscusi ricerca (e infine ritrova) nel suo delicato percorso di ritorno alle origini.

In merito alla scelta di Parigi come luogo "a metà" tra l'America e l'Italia in cui concedersi una sosta prima di raggiungere la meta, avevo molti dubbi. Non riuscivo a capire perché la vicenda di Astoria prendesse avvio proprio da qui, e sempre da qui riaffiorassero i vecchi ricordi dell'autore. Trovo una giustificazione davanti al primo grande parallelismo di tutto il libro, quello tra la defunta madre dell'autore e Napoleone Bonaparte, personaggio che entra a far parte del romanzo perché è proprio davanti alla sua tomba che Viscusi avvia il meccanismo del flashback. La corrispondenza può non essere immediata, ma noi sappiamo che anche il grande condottiero ha origini italiane; è ammesso per questo nella fitta rete di figure e accezioni della storia italiana che Viscusi ripercorre in questa "zona franca" europea dove si reca come visiting professor del Brooklyn College.

Parigi tornerà nei versi di Ellis Island col suo carico di romanticismo, anche questa volta aggravato da un presentimento mortuario e lugubre. Un romanticismo ribaltato nell'immagine dell'arrivo al porto che stravolge le aspettative e promette un futuro infelice: «the couple stands on the quay kissing as if it were paris $|[\ldots]| \mid$ on ellis island begins the dark eternal paris».5

apparirà alla fine del romanzo, nel momento in cui si sarà finalmente riappropriato della sua anima italiana, della sua "storia".

${ }^{4}$ Viscusi, Astoria, p. 20 (trad. it. p. 18: «Aveva quattro nonni. La madre di suo padre. Il patrigno di suo padre. Vivevano a Sunnyside. Poi la madre di sua madre. Il padre di sua madre. Costoro vivevano ad Astoria, un'altra parte del Queens. La chiamavano l'Astoria: inconsapevolmente, un complicato gioco di parole che significava la storia e anche l'astoria. Dal momento che non ci capirono niente, lui aveva il dovere di farlo. Astoria, l'Astoria, era una traccia persistente. Continuò a cercarla ovunque a Parigi. Ma cominciamo dall'inizio»).

${ }^{5}$ Viscusi, Ellis Island 1.1-4.12, sonetto 1.7, vv. 3-5: «la coppia si bacia sul molo come se fosse a parigi | [...] || su ellis island nasce l'eterna parigi scura». 
Gli americani discendenti dagli emigranti italiani avevano dell'Italia un'idea confusa, invasa da stereotipi e pregiudizi, per esempio quella di un Paese prevalentemente agricolo e poco contaminato dal progresso industriale. Robert Viscusi non si vergogna ora a parlare della difficile accettazione delle proprie origini, del "risveglio" avuto in tarda età, del faticoso apprendimento della lingua italiana realizzato fuori tempo massimo: «Non volevo essere italiano, da giovane credevo nel mito americano, avevo questa fede»; e ancora: «Mia moglie voleva fare il viaggio di nozze in Italia [...] allora per proteggermi dall'infezione dell'Italia sono andato prima ad Oxford». Aneddoti di questo tipo, raccontati di persona durante l'incontro di Bologna, possono sembrarci divertenti, ma una volta erano i pensieri fissi di chi non voleva far parte di una determinata cultura e si sentiva marchiato a fuoco da qualcosa che non gli apparteneva. L'esperienza di Robert Viscusi dimostra il progressivo abbandono delle credenze errate nei confronti dei propri antenati e dell'Italia stessa. Attraverso la visita nelle città d'arte gli italoamericani stanno scoprendo lati dell'Italia che conoscevano poco: il ricco patrimonio artistico, la varietà del paesaggio, l'importanza di personaggi storici e letterari di cui forse avevano solo sentito parlare: «Se questa è l'Italia voglio essere italiano!»: è il pensiero di Viscusi davanti alla Certosa di Pavia in occasione di uno dei suoi primi viaggi nel nostro Paese. ${ }^{6}$

Finalmente il passato e il presente si mescolano nell'Astoria (o ad Astoria), forma fisica e concetto che la storia assume per sempre. L'autore stesso non riesce (o forse non vuole) distinguere i due piani temporali, poiché tutto si esaurisce in quest'unica idea, in questa storia a cui Viscusi ha dato il nome di "Astoria": «You understand already, no doubt, what I can't quite keep clear when I'm writing, how la storia and l'Astoria are also the same thing, at least when he speaks of the one as happening in the other $[\ldots] \gg .^{7} \mathrm{Al}$ termine della lettura saremo certamente travolti da quel senso di pace e armonia dato dalla riconciliazione finale avvenuta tra l'autore e un mondo che, con tanta fatica, egli ha imparato ad amare.

\footnotetext{
${ }^{6}$ Per conoscere altri dettagli della storia personale dell'autore si veda anche Danilo Catania, Gianfranco Zucca, Prospettive di una cultura (finalmente) italo-americana: Camaiti Hostert, Carravetta, Tamburri, Viscusi, in «Altreitalie - Rivista internazionale di studi sulle migrazioni italiane nel mondo», 34 (2007), pp. 117-118.

${ }^{7}$ Viscusi, Astoria, p. 31 (trad. it. p. 31: «Comprendete già, indubbiamente, ciò che non riesco affatto a mettere in chiaro mentre scrivo, cioè come la storia e l'Astoria siano anche la stessa cosa, almeno quando si parla di una delle due che si verifica all'interno dell'altra [...]»).
} 